\title{
Comparison of KOH with Culture in the Diagnosis of Dematophytic Fungal Infection in a Tertiary Care Hospital
}

\author{
Shaveta Kataria ${ }^{1 *}$, Shipra Galhotra ${ }^{1}$, Priya Kapoor ${ }^{2}$, Neerja Jindal ${ }^{1}$, Trimaan Kaur Bains ${ }^{1}$ and Rinkal Kansal ${ }^{1}$ \\ ${ }^{1}$ Department of Microbiology, GGS MCH Faridkot \\ ${ }^{2}$ Department of Dermatology and VD, AIIMS Bathinda
}

\begin{abstract}
Skin mycoses also called dermatophytosis is the most common fungal infection affects $20-25 \%$ of World population. Its prevalence varies place to place with climatic changes, time to time with age, sex and lifestyle of the population. Dermatophytoses could be caused by dermatophytes and non-dermatophytes, their frequency of isolation differs with geographical variation.

Objectives: To study various clinical presentations of skin mycosis and compare KOH with culture in the diagnosis of dermatophyticinfections in a tertiary care hospital.

Material \& Methods: A total of 98 skin scrapings collected from consecutive OPD patients from 1st June 2016 to 31 st May 2017 examined using $\mathrm{KOH}$ and culture on modified Sabouraud's dextrose agar medium. The isolated fungi were identified by morphology, lactophenol cotton blue, slide culture and biochemical tests.

Results \& Discussions: T.corporis(40.82\%) was the most common clinical presentation followed by T.cruris(17.35\%), T.pedis(15.31\%), T.capitis $(8.16 \%)$. Fungal infections were demonstrated in 52/98(53.06\%). The male to femaleratio of the positive cases was 15:9. The most affected age group in males $30-40 \mathrm{yrs}$ and females $40-50 \mathrm{yrs}$. $\mathrm{KOH}$ positive were $43.87 \%(43 / 98)$. The samples which were positive in both $\mathrm{KOH}$ and culture were $17.35 \%(17 / 98)$, those positive in $\mathrm{KOH}$ and culture negative were $26.53 \%(26 / 98)$ and $\mathrm{KOH}$ negative and culture positive were $7.14 \%(7 / 98)$. Out of 24 positive cultures, 21 were dermatophytes and threewere non-dermatophytes. The most common dermatophytes were T.mentagrophytes(62.5\%) followed by T.rubrum(20.8\%) and M.gypseum(4.33\%).
\end{abstract}

Conclusion: Skin mycoses is caused mainly by dermatophytes (T.mentagrophytes followed by T.rubrum) but non-dermatophytic infections do occur. Therefore, fungal culture is imperative for correct diagnosis and proper treatment.

Keywords: Dermatophytes, Skin Mycoses, Non-Dermatophytes, Lactophenol Cotton Blue Preparation, Tinea Spp.

\section{Introduction}

Skin mycoses the most common fungal infection affects $20-25 \%$ of World population (WHO 2005). ${ }^{[1]}$ Its prevalence varies from place to place with climatic changes and time to time with age, sex and lifestyle of the population. It is more prevalent in tropical and subtropical countries like India where the heat and humidity are high for most part of the year. Dermatophytes thrive at surface temperatures of $25-28^{\circ} \mathrm{C}$ and infection of human skin is supported by warm and humid conditions. For these reasons superficial fungal infections are relatively common in tropical countries and are exacerbated by the wearing of occlusive clothing. In addition, the frequency of dermatomycoses is greater in communities with low socioeconomic status: crowded living conditions provide multiple opportunities for skin-to-skin contact and close proximity to animals, where hygiene may be suboptimal. Moreover, superficial skin infections show a low tendency to self-limitation and absence of/poor medical care further increases the spread of skin mycoses. ${ }^{[2]}$
Despite regional characteristics and predispositions for dermatophytic infections, the spectrum of dermatophytes is not static. Booming mass tourism, international sports activities and increasing migration mean that less common or forgotten species are being imported and disseminated. ${ }^{[2]}$ Dermatophytoses could be caused by dermatophytes (Genera Trichophyton, Microsporum and Epidermophyton) and non-dermatophytic fungi (Candida, Fusarium, Trichosporon and Scytalidium) and their frequency of isolation also differs with the geographical variation. Over the decades, non-dermatophytes, as agents of superficial fungal infection in humans, produce lesions that are clinically similar to those caused by dermatophytic infections. ${ }^{[3.4]}$ These fungi grow best on those areas of the skin that are warm, dark and moist. These organisms are assuming greater significance due to the excessive use of immunosuppressive drugs for controlling serious as well as non-infectious conditions. They produce keratinases which degrade the keratin and thus, invade the superficial skin tissue. They are usually restricted to non-living cornified layer of the epidermis because of their inability to 
penetrate viable tissue of an immunocompetent host. Acid proteinases, elastase, keratinases and other proteinases reportedly act as virulence factors. ${ }^{[5]}$

Since, these infections are often confused with other skin disorders, it is therefore, necessary to make early laboratory diagnosis for better management of these conditions. The present study was conducted to study the prevalence of different dermatophytic and non-dermatophytic fungi causing skin mycoses in a tertiary care hospital and also done for comparison of $\mathrm{KOH}$ microscopy as compared to culture in early diagnosis of fungal infections.

\section{Material and Methods}

Study design: Descriptive study. Study Area: Department of Microbiology, Tertiary care hospital. Study Population: Patients presenting from skin department of a Tertiary care hospital. Study duration: One year study. Sample Size: On an average 10 skin scrapings/month from skin department are received in the department of Microbiology. As the study period was one year all the skin scrapings had been included in the study. Sampling technique: Nonprobability consecutive sampling technique.

A total of 98 skin scrapings collected from same number of consecutive patients who visited OPD of dermatology and VD department of tertiary care hospital from $1^{\text {st }}$ June 2016 to $31^{\text {st }}$ May 2017 were examined by making $\mathrm{KOH}$ preparation and culture on modified Sabouraud's dextrose agar medium.

The assessment of the participants consisted of an interview, clinical examination and collection of specimens for microbiological studies. All patients completed a questionnaire that contained demographic data, patient and family history and specific data related to risk factor for dermatophytosis and non-dermatophytoses such as age, gender, physical activities, occupation, predisposing diseases and contact with animals and clinical diagnosis.

Sample collection and transportation: the suspected site was cleaned with $70 \%$ ethyl alcohol and material was transported in black paper and transferred to the laboratory as soon as possible for direct microscopic examination and culture.

Microscopic examination of specimens: was performed with an aqueous solution of $20 \%$ potassium hydroxide $(\mathrm{KOH})$ mixed with $5 \%$ glycerol.

Culture: All specimens were cultured on modified Sabouraud's dextrose agar medium (SDA along with chloramphenicol and cycloheximide), both with and without antibiotics and incubated at $25^{\circ} \mathrm{C}, 37^{\circ} \mathrm{C}$ for 21 days.
Examination and identification of fungus isolates: The isolated fungi were identified by morphology, lactophenol cotton blue preparation, slide culture and biochemical tests (hair perforation test, urease test and growth characteristics on bromocresol purple agar).

The study was approved by authorisation committee of our hospital and was carried out in accordance with previously reported recommendations on the design and conduct of diagnostic accuracy assessments.

\section{Statistical analysis}

The data pertaining to sociodemographic and other clinical variables was entered in the form of data matrix in Microsoft ${ }^{\circledR}$ Excel $^{\circledR}$ and analysed using IBM $^{\circledR} \operatorname{SPSS}^{\circledR} \mathrm{v}$ 20.0.0. The descriptive statistics for categorical variables was represented in the form of frequencies and percentages and as means and standard deviations for continuous variables.

\section{Results}

Out of the total 98 clinically suspected specimens, the most common infections were Tinea corporis 40 (40.8\%), followed by Tinea cruris 17 (17.35\%), Tinea pedis 15 (15.3\%), Tinea capitis 8 (8.2\%), Tinea cruris et corporis 4 (4.1\%), Recurrent Tinea, Tinea facei and Tinea mannum $3(3.1 \%)$ each, Tinea cruris et pedis and Tinea incognita 2 (2\%) each and Tinea mannum et pedis 1 (1\%) (Figure 1). Figure 7 shows erythematous patches with active borders near shoulder and groins. Out of 98 clinically suspected cases of skin mycoses, 58 were males and 40 were females (Figure 2). Fungal infections were demonstrated in 52/98(53.06\%) (Figure 3,4). The male to female ratio of the positive cases were recorded as 15:9. $\mathrm{KOH}$ positive samples were $43.87 \%$ (43/98). The samples which were positive in both $\mathrm{KOH}$ and culture were $17.35 \%$ (17/98), those positive in $\mathrm{KOH}$ preparation and culture negative were $26.53 \%$ (26/98) and $\mathrm{KOH}$ negative and culture positive were $7.14 \%(7 / 98)$ (Figure 5). The most affected age group in males were 30-40yrs and in females 40-50yrs (Table 1). Out of 24 positive cultures, 21 were dermatophytes and three were non-dermatophytes. The most common dermatophytes were T. mentagrophytes $(62.5 \%)$ followed by T. rubrum (20.8\%) and M. gypseum (4.33\%) (Figure 6). Figure 8- Trichophyton mentagrophytes showing spiral hyphae and clusters of microconidia. (LCB * 400). T. corporis was found in 40 clinically suspected cases and out of which 24 were $\mathrm{KOH}$ positive and on culture 6 isolates were T. mentagrophytes, 4 were T. rubrum and one isolate was Fusarium sp. T. cruris were found in 17 clinically suspected cases, 7 were $\mathrm{KOH}$ positive for fungal infections and $4 T$. mentagrophytes and one isolate was $T$. rubrum (Table 2). Culture is gold standard test done for fungus 
but other biochemical tests are helpful in identification of fungus. Sensitivity, specificity, PPV and NPV of KOH in comparison to Culture were $70.83 \%, 62.16 \%, 37.78 \%$ and $86.8 \%$ ( $\mathrm{p}=0.0017$ test is significant) (Table 3 ).

Table 1: Age wise distribution of clinically suspected cases in relation to culture $(n=98)$.

\begin{tabular}{|c|c|c|}
\hline AGE IN YEARS & $\begin{array}{c}\text { MALE- TOTAL } \\
\text { (CULTURE POSITIVE) }\end{array}$ & $\begin{array}{c}\text { FEMALE- TOTAL } \\
\text { (CULTURE POSITIVE) }\end{array}$ \\
\hline $0-10$ & $3(0)$ & $2(1)$ \\
\hline $11-20$ & $6(2)$ & $2(1)$ \\
\hline $21-30$ & $21(3)$ & $7(1)$ \\
\hline $31-40$ & $11(4)$ & $7(2)$ \\
\hline $41-50$ & $8(3)$ & $13(3)$ \\
\hline $51-60$ & $4(2)$ & $7(1)$ \\
\hline $61-70$ & $4(1)$ & $2(0)$ \\
\hline $71-80$ & $0(0)$ & $0(0)$ \\
\hline $81-90$ & $1(0)$ & $0(0)$ \\
\hline TOTAL & $58(15)$ & $40(9)$ \\
\hline
\end{tabular}

Table 2: Comparison of types of skin mycosis and Culture results $(n=98)$.

\begin{tabular}{|c|c|c|c|}
\hline Types of skin mycosis & $\begin{array}{c}\text { Total clinically suspected } \\
\text { cases }\end{array}$ & KOH positive & Fungus isolates \\
\hline T. corporis & 40 & 24 & $4 \mathrm{~m}^{*}, 4 \mathrm{r}^{* *}, 1 \mathrm{f}^{* * *}$ \\
\hline T. cruris & 17 & 7 & $3 \mathrm{~m}^{*}, 1 \mathrm{r}^{* *}, 1 \mathrm{a}^{* * * * *}$ \\
\hline T. pedis & 15 & 0 & 0 \\
\hline T. capitis & 8 & 2 & $1 \mathrm{~m}^{*}$ \\
\hline T. cruris et corporis & 4 & 1 & $1 \mathrm{~m}^{*}$ \\
\hline Recurrent tinea & 3 & 1 & $1 \mathrm{~g}^{* * * *}$ \\
\hline T. mannum & 3 & 0 & 0 \\
\hline T. facei & 3 & 1 & $1 \mathrm{a}^{* * * * *}$ \\
\hline T. cruris et pedis & 2 & 0 & 0 \\
\hline T. incognita & 2 & 0 & 0 \\
\hline
\end{tabular}

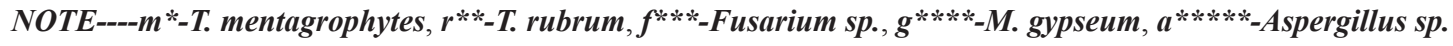

Table 3: The results of various biochemical tests performed for identification of dermtophytes.

\begin{tabular}{|l|l|l|l|}
\hline & Hair perforation test & Urease test & Bromocresol purple agar \\
\hline T. mentagrophytes & Positive & Positive & Alkaline \\
\hline T. rubrum & Negative & Negative & Alkaline \\
\hline M. gypseum & Positive & Positive & No change \\
\hline
\end{tabular}

Table 4: Showing sensitivities and specificities of $\mathrm{KOH}$ in comparison to Culture.

\begin{tabular}{|l|l|l|l|l|}
\hline CULTURE POSITIVE NEGATIVE TOTAL & \multicolumn{1}{l|}{} \\
\hline \multirow{3}{*}{$\mathrm{KOH}$} & POSITIVE & 17 & 26 & 43 \\
\cline { 2 - 6 } & NEGATIVE & 07 & 48 & 55 \\
\cline { 2 - 5 } & TOTAL & 24 & 74 & 98 \\
\hline
\end{tabular}

Statistically significance (McNemar's Test) p value =0.0017 (Statistically significant) Sensitivity 70.83\% [95\% CI 48.91-87.38\%], Specificity 64.86\% [95\% CI 52.89-75.61\%], PPV 39.53\% [95\% CI 30.43-49.43\%] NPV 87.27\% [95\% CI 78.24-92.90\%]. 


\section{Clinicaly suspected fungal infections}

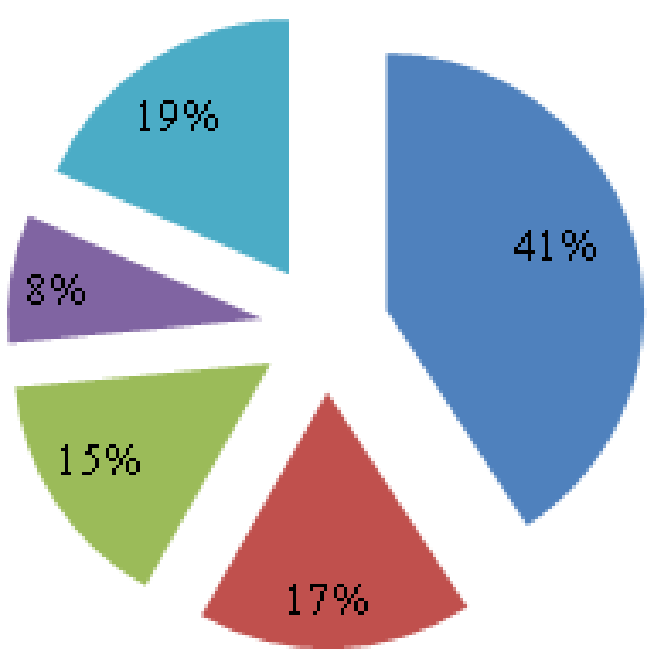

T.CORPORIS

T.CRURIS

T.PEDIS

-T.CAPITIS

OTHERS

Fig. 1: Pie chart showing distribution of clinically suspected fungal infections $(n=98)$.

\section{Clinically suspected cases}

"MALE $\quad$ FEMALE

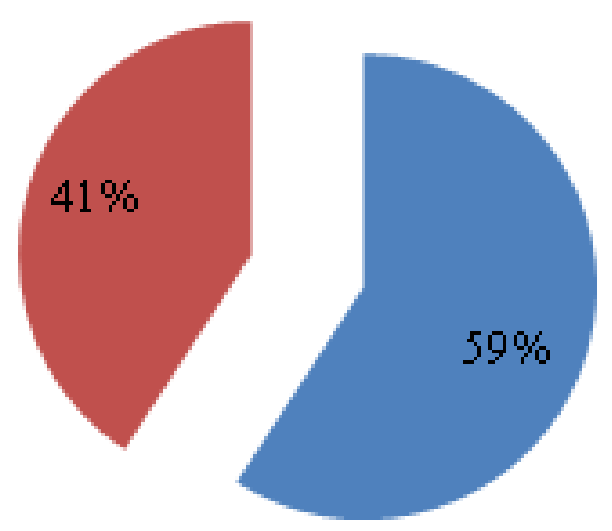

Fig. 2: Pie chart showing sex wise distribution of clinically suspected cases. 


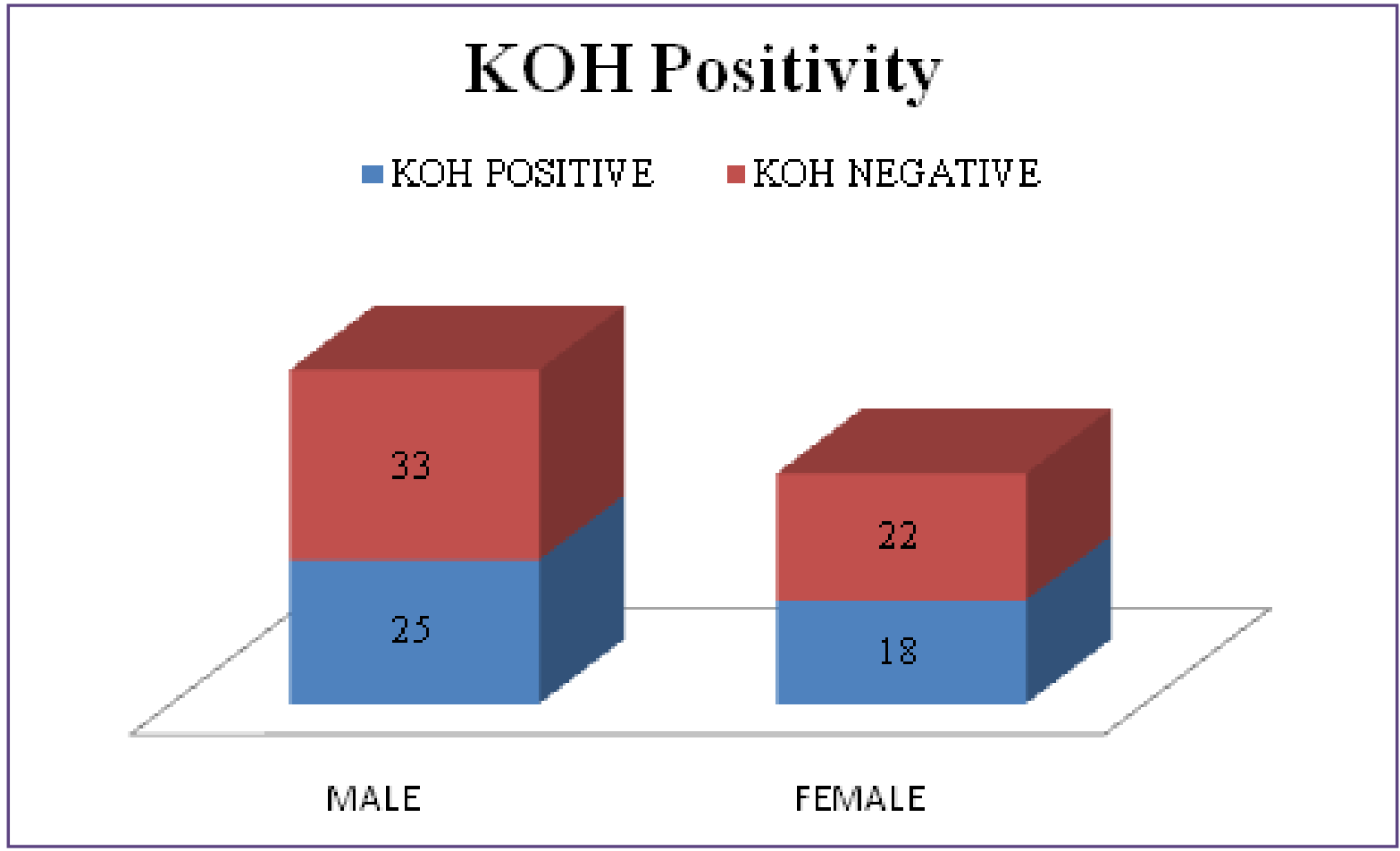

Fig. 3: Histogram showing КОH test results (n=98)..

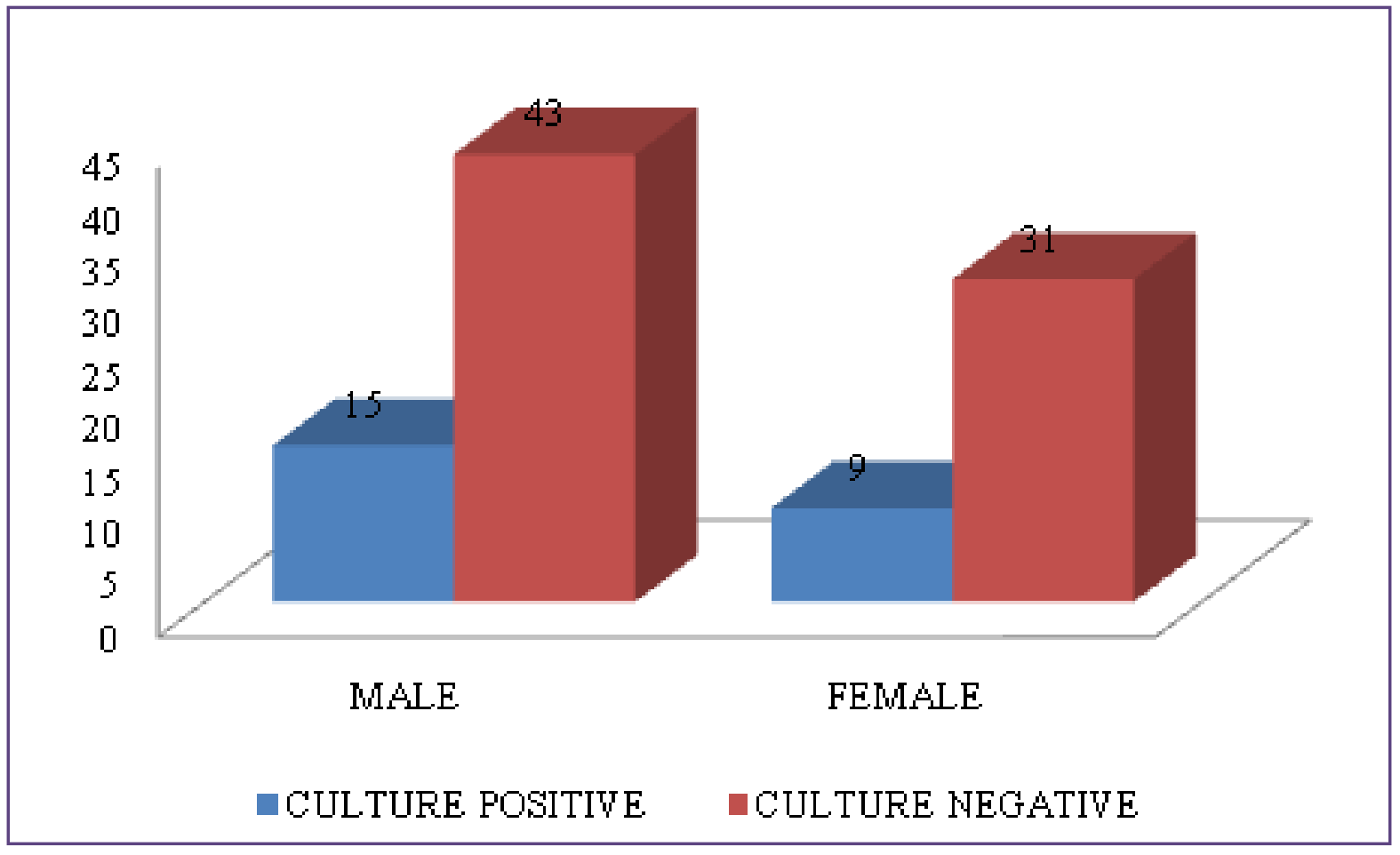

Fig. 4: Histogram showing gender wise incidences in total of 98 patients under study. 


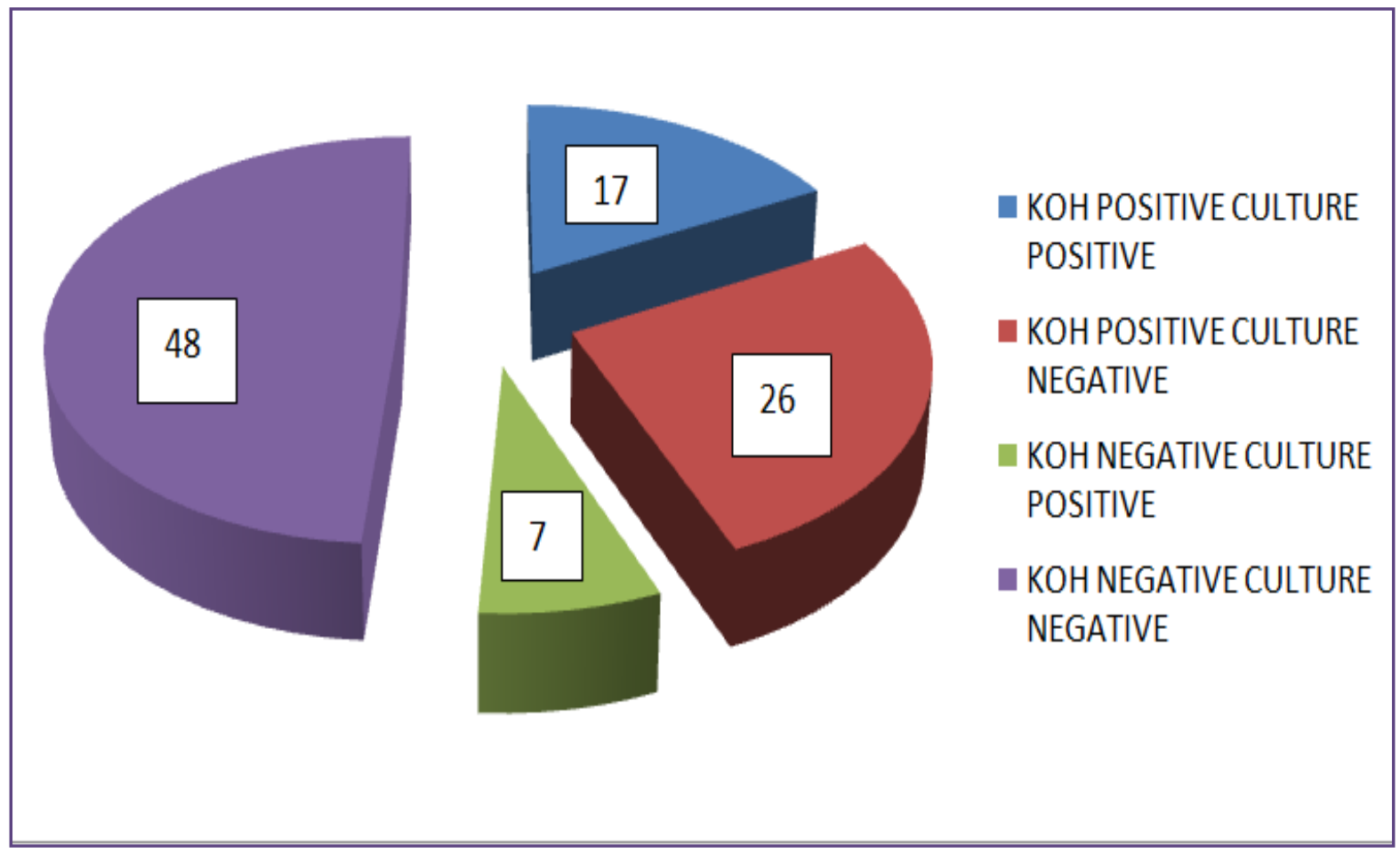

Fig. 5: Pie chart showing sensitivity of KOH test verses culture test of 98 case study.

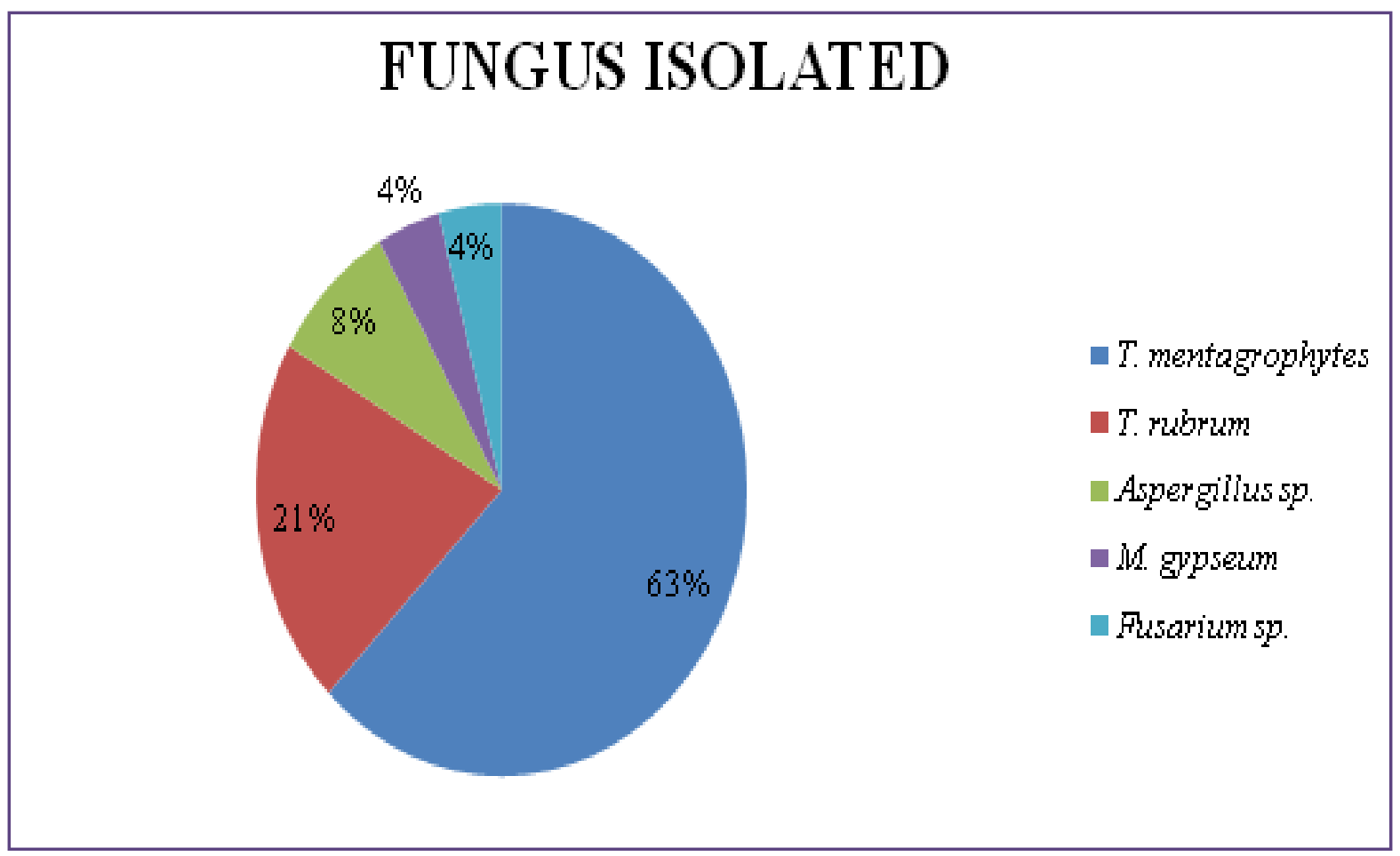

Fig. 6: Various fungus isolated on culture $(n=24)$. 


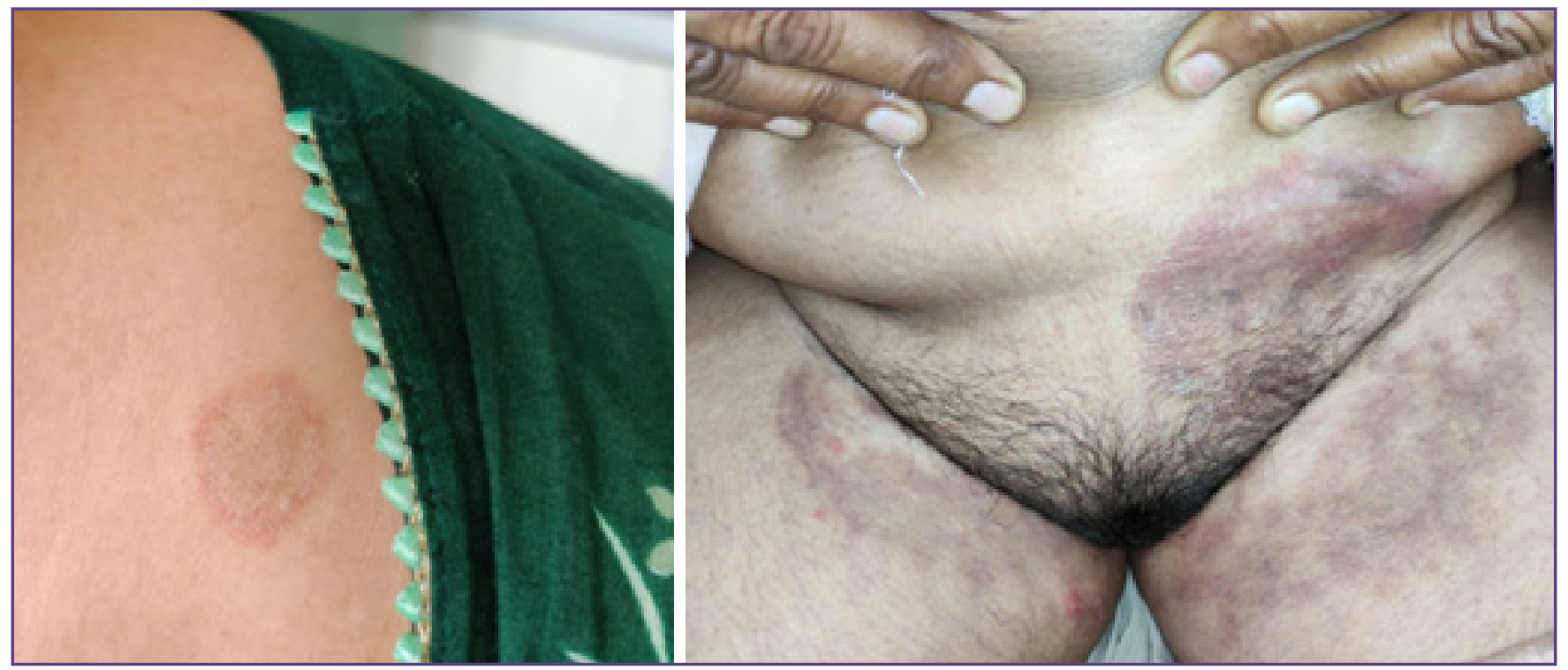

Fig. 7: shows erythematous patches with active borders near shoulder and groins.

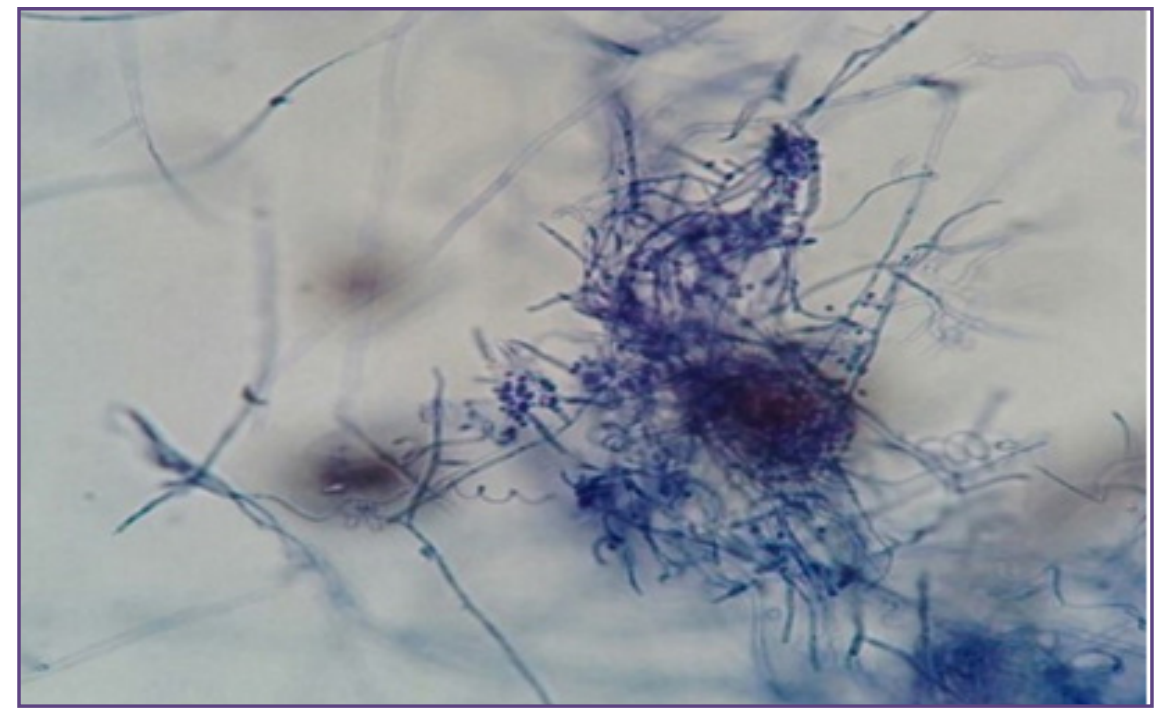

Fig. 8: Trichophyton mentagrophytes showing spiral hyphae and clusters of microconidia. (LCB * 400).

\section{Discussion}

Although any outbreak or pandemic has not been reported due to dermatophytes but incidence of severe systemic fungal infections had increased significantly. The cause of this could be excessive or irrational use of antibiotics, steroids and many other clinical disorders that makes the individual immunocompromised. The present study was done to determine the prevalence of skin mycosis caused by dermatophytic and other non-dermatophytic fungi.

The prevalence of dermatophytes among fungal skin infections was found to be $87.5 \%$. Nasimuddin et al has shown slightly higher prevalence and Bitew et al has shown lower prevalence $(69.8 \%){ }^{[6,7]}$ In our study, T. corporis $(40.8 \%)$ was the most common clinical presentation followed by $T$. cruris $(17.35 \%)$, T. pedis $(15.31 \%), T$. capitis $(8.16 \%)$ (Figure 1$)$. This is in concordance with the various studies by Bhatia VC et al, Sowmya $\mathrm{N}$ et al and Gupta $\mathrm{S}$ et al. ${ }^{[1,2,3]}$ While the study conducted by Elmegeed et al showed that the T. capitis $(28.6 \%)$ was the most common, followed by $T$. pedis $(21.21 \%)$ and $T$. corporis $(14.8 \%) .{ }^{[4]}$ A study conducted on 67 Iraq patients (2020) with various dermatophytosis infections, T. pedis $(45.1 \%)$ was the most common followed by T. mannum $(22.2 \%), \mathrm{T}$. capitis (11.8\%), T. corporis (7.8\%), T. ungium (5.9\%). ${ }^{[8]}$ 
Among the suspected patients there were more infections in males as compared to females (Figure 2). This correlates with other studies. ${ }^{[9,10,11]}$ This may be due to the differences in occupational exposure as males are more involved in construction and other works. Among the patient data collected, out of 58 males, 25 were $\mathrm{KOH}$ positive and 18 out of 40 females were $\mathrm{KOH}$ positive (Figure 3). Similar observations were recorded at the National Skin Centre, a tertiary referral centre for dermatological diseases in Singapore where majority of patients $(n=9335)(72.3 \%)$ were males. This was also seen in anthropophilic species, which is the main cause of dermatophytosis in adults. ${ }^{[5]}$

Among the patient data collected, the total culture positivity was $24.5 \%$ (24/98) (Figure 4). Similar findings were observed by Gupta et al while Sowmya et al reported much higher $(49 \%)$ culture positivity. ${ }^{[2,3]}$

Out of the 98 specimens, $\mathrm{KOH}$ positive samples were 43 (43.87\%) while culture positive were 24 (24.49\%). The samples which were positive in both $\mathrm{KOH}$ and culture were $17.35 \%$ (17/98), those positive in $\mathrm{KOH}$ preparation and culture negative were $26.5 \%$ (26/98) (false positive) and $\mathrm{KOH}$ negative and culture positive were $7.14 \%(7 / 98)$ (false negative) (Figure 5). Similar results were observed in the study conducted by Gupta et al. ${ }^{[3]}$

The most common age group among males was 21-30 years and 41-50 years among females in relation to culture (Table 1). The probable reason for higher prevalence in this group could be that the individuals in this group are often most active because of their involvement in the outdoor activities such as studies, jobs etc. While the study conducted by Elmegeed et al showed that the most common age group was 0 -10years followed by $11-20$ years. ${ }^{[4]}$ This might be because of more population of low economic status which maintain poor personal hygiene.

Out of 24 positive cultures, $21(87.5 \%)$ were dermatophytes and $3(12.5 \%)$ were non-dermatophytes, while that of Appalaraju B et al dermatophytes $43 \%$ isolated and non-dermatophytes were $6 \%{ }^{[2]}$ The most common dermatophytes were T. mentagrophytes $(62.5 \%)$ followed by $T$. rubrum (20.8\%) and M. gypseum (4.33\%) which is in concordance with the results from other studies (Figure 6). ${ }^{[1,12]}$ In Europe there was higher incidence of T. rubrum infections was recorded while in Asia T. mentagrophytes was the commonest isolate. ${ }^{[8]}$

The most common isolate from the clinical samples in our study was T. mentagrophytes, followed by T. rubrum and Fusarium sp (Table 2). The reason for more prevalence of T. mentagrophytes in our study could be due to more labour class population with increased migration and climatic changes.

The biochemical tests were done to differentiate among dermatophytes. T. mentagrophytes and M. gypseum were both hair perforation and urease test positive but both differ on bromo cresol agar. T. rubrum both hair perforation and urease test negative (Table 3 ).

Culture is gold standard test done for fungus but other biochemical tests are helpful in identification of fungus. Sensitivity, specificity, PPV and NPV of $\mathrm{KOH}$ in comparison to Culture were $70.83 \%, 62.16 \%, 37.78 \%$ and $86.8 \%$ ( $P=0.0017$ test is significant $)$. While study conducted in Jaipur showed that the sensitivity of $\mathrm{KOH}$ was slightly higher $(84 \%)$ as compared to reference standard culture test than our study. ${ }^{[3]}$

The clinical presentation though typical of ringworm infection is very often confused with other skin disorders particularly due to topical application of steroid ointments and creams, leading to further misdiagnosis and mismanagement. ${ }^{[13]}$ Hence there arises a need for correct, efficient and rapid laboratory diagnosis of dermatophytes. With the proper techniques various species can be identified. And it's the right time to look for the molecular approaches for rapid dermatophytes identification.

\section{Conclusion}

Skin mycoses is caused mainly by dermatophytes (T. mentagrophytes followed by T. rubrum) but nondermatophytic infections do occur. Therefore, fungal culture is imperative for correct diagnosis and proper treatment. The data revealed the $\mathrm{KOH}$ test was false positive in 26 cases and false negative in 07 cases studied. Since, the sensitivity of $\mathrm{KOH}$ test is significantly high with reference to culture test, it can be used as a definitive procedure for screening and diagnosis of dermatophytic infection. $\mathrm{KOH}$ test ensures cost effectiveness and require only microscopic facility with trained professionals. It can be recommended that if proper training is provided to the technical staff, precise diagnosis can be established with limited lab facilities even at remote places.

\section{Abbreviations and Symbols}

SDA- Sabouraud's dextrose agar medium, Tinea corporisT. corporis, Tinea cruris- T. cruris, Tinea pedis- T. pedis, Tinea capitis- T. capitis, Tinea cruris et corporis- T. cruris et corporis, Tinea facei- T. facei and Tinea mannum- T. mannum, Tinea cruris et pedis- T. cruris et pedis, Tinea incognita-T. incognita, Tinea mannum et pedis- T. mannum et pedis, Trichophyton mentagrophytes- T. mentagrophytes, Trichophyton rubrum- T. rubrum and Microsporum gypseum- M. gypseum. 


\section{Acknowledgement}

We express our sincere thanks and profound sense of gratitude and indebtness to our eminent teacher, a colossal professional Professor and Head, Department of Microbiology in our college for giving us excellent guidance in this study. This study bears imprint of her vast experience, invaluable suggestions and continuous encouragement at every step of my study. It has been matter of great pride and privilege for us to work under the able guidance of our renowned teacher. A special thanks to Assistant professor, Department of Social and Preventive Medicine for all his help and contribution in statistical analysis.

\section{Funding}

None

\section{Competing Interest}

None

\section{References}

1. Bhatia VK, Sharma C. Epidemiological studies on dermatophytosis in human patients in Himachal Pradesh, India. Springers Plus. 2014;3:134.

2. Sowmya N, Appalaraju B, Surendran P, Srinivas CR Isolation, identification and comparatative analysis of SDA and DTM for dermatophytes from clinical samples in a tertiary care hospital. IOSR-IJDS. 2014;13(11):68-73.

3. Gupta S, Agrwal P, Rajawat R, Gupta S. Prevalence of dermatophytic infections and determining sensitivity of diagnostic procedures. IJPPS. 2014;6(3):35-8

4. Elmegeed Al-SM, Ouf SA, Moussa TAA, Eltahlawi SMR. Dermatophytes and other associated fungi in patients attending to some hospitals in Egypt. Braz J Microbiol. 2015;46(3):799-805.

5. Svejgaard E. Epidemiology and clinical features of dermatomycoses and dermatophytoses. Acta Derm Venereo. 1986;12(1):19-26.

6. Nasimuddin S, Appalaraju B, Surendran P, Srinivas CR. Isolation, identification and comparatative analysis of SDA and DTM for dermatophytes from clinical samples in a tertiary care hospital. J Dental Med Sci. 2014;13(11):68-73.

7. Bitew A. Dermatophytosis: prevalence of dermatophytes and non-dermatophyte fungi from patients attending arsho advanced medical laboratory, Addis Ababa, Ethiopia. Dermatol Res Prac. 2018;8164757:1-6.

8. AL-Khikani FHO. Dermatophytosis a worldwide contagious fungal infection: growing challenge and few solutions. BBRJ. 2020;4(2):117-22.

9. Singh S, Beena PM. Profile of dermatophyte infections in Baroda. Indian $\mathrm{J}$ Dermatol Venereol Leprol. 2003;69(4):281-3.

10. King-man HO, Cheng T. Common superficial fungal infections- a short review. Hong Kong Med Diary. 2010;11:23-7.

11. Balakumar S, Rajan S, Thirunalasundari $\mathrm{T}$, Jeeva $\mathrm{S}$ Epidemiology of dematophytosis in and around Tiruchipalli, Tamil Nadu, India. Asian Pac J Trop Dis. 2010;31(3):295-8.

12. Aggarwal A, Arora U, Khanna S. Clinical and mycological study of superficial mycoses in Amristar. Indian J Dermatol. 2002;47:218-20.

13. Burzykowski T, Molenberghs G, Abeck D, Haneke E, Hay R, Katsambas A, et al. High prevalence of foot diseases in Europe: results of the Achilles Project. Mycoses. 2003;46:496-505.

*Corresponding author:

Dr. ShavetaKataria, H. No. 70 New Green Avenue colony OppRose garden Bathinda, Punjab 151001

Phone: +91 9646161331

Email: dimcikataria.25@gmail.com

Date of Submission $\quad:$ 24/12/2020

Date of Final Revision : 04/03/2021

Date of Acceptance $\quad$ : 09/04/2021

Financial or other Competing Interests: None.

Date of Publication $\quad: 30 / 04 / 2021$ 